\title{
THE POLYCHROMATOR: A PROGRAMMABLE MEMS DIFFRACTION GRATING FOR SYNTHETIC SPECTRA
}

\author{
G. B. Hocker, D. Youngner \\ Honeywell Technology Center \\ Plymouth, MN 55441-4799
}

\author{
M. Butler, M. Sinclair, T. Plowman \\ Sandia National Laboratories \\ Albuquerque, NM 87185
}

\begin{abstract}
We report here the design, fabrication and demonstration of an electrostatically actuated MEMS diffractive optical device, the Polychromator grating. The Polychromator grating enables a new type of correlation spectrometer for remote detection of a wide range of chemical species, offering electronic programmability, high specificity and sensitivity, fast response and ruggedness. Significant results include: (1) The first demonstrations of userdefined synthetic spectra in the $3-5 \mu \mathrm{m}$ wavelength regime based upon controlled deflection of individual grating elements in the Polychromator grating; (2) The first demonstration of gas detection by correlation spectroscopy using synthetic spectra generated by the Polychromator grating.
\end{abstract}

\section{INTRODUCTION}

MEMS technology enables many innovative optical devices. The Polychromator grating is an array of a large number of long, narrow optically reflective elements whose vertical positions are electrically controlled. If the element length is many optical wavelengths, the width a few optical wavelengths, and vertical positions controllable to a fraction of a wavelength, the Polychromator can function as a programmable diffractive optical device. Specifically, as described below, the Polychromator grating elements can be positioned to generate a desired multi-line, or polychromatic, spectrum at a given diffraction angle. Furthermore, this synthetic spectrum can be designed to match the infrared absorption spectrum of a molcculc and used in a correlation spectrometer for the spectroscopic detection of that species.

\section{MEMS DEVICE DESIGN}

The Polychromator gratings reported here are designed to generate synthetic spectra in the $3-5 \mu \mathrm{m}$ wavelength range. A grating consists of 1024 individually addressable diffractive elements each $10 \mu \mathrm{m}$ wide by $1 \mathrm{~cm}$ in length. The vertical position of a grating element is to be controlled over approximately a $2 \mu \mathrm{m}$ range with an accuracy of about $0.1 \mu \mathrm{m}$. Because of the difficulty of providing individual inputs to 1024 lines, the first devices fabricated used only 132 pads for signal and ground, compatible with a readily available pin-grid-array packagc. In order to actuate all 1024 lines with this limited 1/O capability, the grating elements are hard-wired on-chip as 8 repeat units of 128 elements, such that a voltage applied to a pad actuates 8 lines, one from each repeat unit. This on-chip hard wiring, while advantageous for device packaging and initial testing, has a deleterious effect on device yield, since if any one of the eight lines connected to a pad is compromised, the entire set of 8 lincs is non-functional.

\author{
E. Deutsch, A. Volpicelli, S. Senturia \\ Massachusetts Institute of Technology \\ Cambridge, MA 02139
}

\author{
A. J. Ricco \\ ACLARA BioSciences \\ Mountain View, CA 94043
}

Nevertheless, successful results have been obtained using this configuration.

The deflection of each element is a function of the applied voltage, and the maximum travel is limited by an electromechanical instability called pull-in. For the beam lengths and widths used in this design, and with beam thickness comparable to the free-space gap, actuation via a continuous underlying electrode would result in pull-in occurring at approximately two-thirds of the free-space gap. In order to achieve positioning beyond the pull-in instability, the design for the Polychromator device takes advantage of leveraged bending[1]. The element design shown in Figures 1 and 2 uses a two-beam structure attached periodically to the substrate, in which the lower beam undergoes bending while the top mirror beam deflects vertically without flexion. Leveraged bending of the lower beam by voltage applied to the split actuation electrodes located near the support posts allows controllable positioning of the upper mirror beam through the full gap. Electromechanical analysis is used to design structures capable of near-full-gap actuation with voltages of about 100 volts or less. The gaps and beam thicknesses are about $2 \mu \mathrm{m}$. For the devices reported here, post-to-post spacings are in the range of 500 to $700 \mu \mathrm{m}$, and electrode lengths are in the range of 80 to $100 \mu \mathrm{m}$.

\section{DEVICE FABRICATION}

The Polychromator grating device is fabricated by polysilicon surface micromachining. Two polysilicon layers are used for the actuated structure, and two for electrical interconnections and actuating electrodes. The process begins by growing a thermal oxide on an ultra-flat silicon wafer. Two layers of boron-doped polysilicon separated by silicon nitride are used for the interconnection and electrode structures. Next a relatively thick sacrificial $\mathrm{SiO}_{2}$ layer is deposited, which will define the actuation gap. Support posts for the bending layer of polysilicon are patterned and etched in this $\mathrm{SiO}_{2}$ layer. Before depositing the bending layer of polysilicon, small "dimples" are etched in the $\mathrm{SiO}_{2}$. The dimples cause nodules to form on the bottom of the polysilicon, and the nodules prevent stiction during operation should the bending beam pull in. The bending layer of polysilicon is deposited, implanted, and patterned, followed by the deposition of the second sacrificial $\mathrm{SiO}_{2}$ layer. The second $\mathrm{SiO}_{2}$ layer has vias etched in it to create support posts for the top polysilicon mirror beam layer. The top polysilicon mirror-beam layer is then deposited, implanted and patterned. Typical thicknesses for the bending and mirror beam layers of polysilicon and for the two sacrificial $\mathrm{SiO}_{2}$ layers are approximately $2 \mu \mathrm{m}$. Next the wafers are anncalcd to set the polysilicon stress. The process is completed by depositing gold for the mirrors and pads, then removing all of the sacrificial $\mathrm{SiO}_{2}$. The fabricated element and grating are shown in Figures 3,4 , and 5 . 


\section{GRATING ACTUATION RESULTS}

The individual diffractive elements of the Polychromator grating are actuated electrostatically. Beam deflection depends on the applied voltage, electrode length, distance between support posts, beam thickness, residual stress, and free-space gap. Combinations of mask design and process parameters are selected to result in structures which maximize travel while maintaining moderate actuation voltage. The vertical position of each element is determined by the applied voltage. To evaluate fabricated devices, interference microscopy is used to measure vertical movement of individual beam elements as shown in Figure 6. Using this technique, the deflection of each beam is measured as a function of applied voltage. Results for several grating element designs are shown in Figure 7. Measured maximum beam deflections for these devices are nearly the full gap of two microns, and pull-in occurs at voltages ranging from 65 to 110 volts, depending on device designs.

\section{OPTICAL OPERATION AND SYNTHETIC SPECTRA}

When infrared radiation strikes a grating, the phase shift of the wavefront on reflection from a given diffractive element depends upon vertical position of that element. The diffracted radiation is the sum of the wavefronts diffracted from the individual grating elements. Since the vertical position of the elements of the Polychromator grating can be controlled by the voltages applied, it is possible to program the Polychromator grating to diffract infrared radiation in a desired manner. A spectral design algorithm has been developed that calculates the displacement profile for the diffractive elements that will generate desired spectra at a specified angle[2]. This algorithm can be used to design novel diffraction gratings that are complex spectral filters with any spectral content desired. They can be used to synthesize the absorption spectra of specific molecules [3] and to function as the reference spectra in a programmable correlation spectrometer [2]. More complex spectral designs can be used to enable realtime multi-variate analysis which can distinguish between chemical species with overlapping spectra. This development extends the application of diffractive optics from spatial-domain optical processing into the spectral domain.

For the Polychromator grating, the spectral design algorithm was used to design the deflection profiles required to produce desired spectra in the $3-5 \mu \mathrm{m}$ wavelength regime. Using measured voltage/displacement data for the grating such as those shown in Figure 7, the deflection profile was converted to a set of voltages that are applied to the groups of elements.

We have for the first time generated synthetic spectra with a programmable MEMS diffraction grating. Two such designed spectra in the infrared are shown in Figure 8; their designs and use in detection of $\mathrm{CO}_{2}$ will be described in the following section.

\section{CORRELATION SPECTROSCOPY AND GAS DETECTION}

A correlation spectrometer correlates the spectrum from a sample with a reference spectrum, providing sensitivity and specificity, fast response, and mechanical and optical simplicity. The need for a reference cell containing a sample of the target species limits its usefulness. It is awkward to change target species by changing the reference cell, and the technique is impractical for transient or highly toxic chemical species. In addition, a large amount of background light is transmitted in spectral regions with no optical absorption, adding to the background level without contributing to the desired signal. Use of the Polychromator grating to generate the reference spectrum enables programmable detection of specific gases species including transient and toxic species. Furthermore, the Polychromator grating can be programmed to produce the complement of the optical absorption spectrum of the target species to be detected. This removes the light transmission at frequencies with no optical absorption, thereby enhancing the signal-to-background ratio.

The optical layout for the correlation spectrometer is shown in Figure 9 . The infrared source is an $1100 \mathrm{~K}$ black body with the light passing through a $10 \mathrm{~cm}$ gas cell. The light is collected by a $\mathrm{CaF}_{2}$ lens and focused on the entrance slit. Subsequently, the light is recollimated by a spherical mirror and directed onto the Polychromator surface. The diffracted light is collected at the desired angle by another flat mirror and then focused on the exit slit. The detector is a liquid-nitrogen-cooled indium antimonide detector (EG\&G Optoelectronics). A high-voltage analog driver instrument (HVADI), custom designed by Digital Designs and Systems, Inc. (dideas.com), serves as the drive electronics for the Polychromator. The HVADI provides 128 control lines, each capable of delivering $0-200$ volts. The HV $\Lambda$ DI's microprocessor and RS-232 port allow for control of the instrument and for uploading of files to generate various grating profiles. The HVADI can modulate the Polychromator by switčhing between two such profiles - a requirement for correlation spectroscopy.

The rate of switching between the two grating profiles can be varied. A flat response is observed for frequencies below $2 \mathrm{kHz}$ with roll-off at higher frequencies. Since the resonant frequency of the beams is in the tens of $\mathrm{kHz}$ range, we believe the roll-off is associated with either an RC time constant formed by the capacitance of the beam and the resistance of the lead providing the voltage, or the current capacity of the instrument driving the beams.

The two synthetic spectra shown in Figure 8 were designed for the detection of $\mathrm{CO}_{2}$. The solid line is a bandpass centered on the $\mathrm{CO}_{2}$ absorption band at $2350 \mathrm{~cm}^{-1}$, while the dashed line is a double bandpass that samples bands on either side of the $\mathrm{CO}_{2}$ absorption line. The integrated intensity of the transmitted light for the two spectra is designed to be equal. Switching between these two profiles generates a modulated signal only if $\mathrm{CO}_{2}$ is present in the gas cell. The modulated output from the detector when the gas cell was alternately filled with pure nitrogen and 1000 ppm of $\mathrm{CO}_{2}$ in nitrogen is shown in Figure 10. This is the first demonstration of gas detection by correlation spectroscopy using synthetic spectra generated by the programmable Polychromator grating.

The fact that the 1024 beam elements are hard wired in 128 groups of eight lines has two important effects on device operation. First, as already stated, there is a reduction in device yield. The results shown here are from a grating with only $25 \%$ of the sets of 8 beams functional. The fact that successful synthetic spectra and chemical detection could be performed with such a device demonstrates the robustness of the Polychromator against localized defects. When all 1024 lines can be independently actuated, functional beam yield will increase significantly because an isolated fault will no longer affect 8 beams. It is estimated that the diffraction efficiency increases as the square of the number of functional elements. Thus, a considerable improvement in performance is expected for a completely functional device.

A second effect of the hard wiring is that the periodicity imposes a sampling in wavelength space for the synthetic spectra, limiting somewhat the spectral features that can be implemented. 
This limitation will be removed when a packaging scheme capable of driving all 1024 lines is implemented.

\section{CONCLUSIONS}

We have demonstrated the Polychromator, a programmable MEMS diffraction grating capable of generating synthetic spectra. The innovative electromechanical design of the electrostatically controlled grating elements uses leveraged bending to allow controllable actuation over the nearly the full gap. Fabrication utilizes multilevel polysilicon surface micromachining. A design algorithm is used to calculate the displacements of the grating elements that will create a desired diffraction spectrum, along with the voltages required to create these displacements. Synthetic spectra have been generated as designed in the $3-5 \mu \mathrm{m}$ wavelength regime. Two such designed spectra have been used to detect $\mathrm{CO}_{2}$ in the first demonstration of correlation spectroscopy using synthetic reference spectra generated by a programmable MEMS device.

The Polychromator concept will lead to an instrument for stand-off detection of many chemical species. Advantages include electronic programmability, specificity to the desired species and immunity to interference, and fast response, in a miniature, rugged, low-power instrument.

Future efforts will include improvements to grating element yield to improve diffraction efficiency, and development of Polychromator gratings and control electronics offering individual control of 1024 grating elements for improved spectral resolution.

\section{ACKNOWLEDGEMENTS}

Support for this research was provided by the Defense Advanced Research Projects Agency under contract N66001-97-C8620. Sandia is a multi-program laboratory operated by Sandia Corporation, a Lockheed Martin Company, for the United States Department of Energy under Contract DE-AC04-94AL85000. The authors would like to acknowledge Robert C. Sanchez for his assistance with the $\mathrm{CO}_{2}$ detection measurements.

\section{REFERENCES}

1. E. S. Hung, S. D. Senturia, "Extending the Travel Range of Analog-Tuned Electrostatic Actuators," Journal of Microelectromechanical Systems, 8, 497 (1999).

2. M. B. Sinclair, M. A. Butler, A. J. Ricco and S. D. Senturia, "Synthetic Spectra: A Tool for Correlation Spectroscopy," Applied Optics, 36, 3342 (1997).

3. M. B. Sinclair, M. A. Butler, S. H. Kravitz, W. J. Zubrzycki and A. J. Ricco, "Synthetic Infrared Spectra," Optics Letters, 22, 1036 (1997).

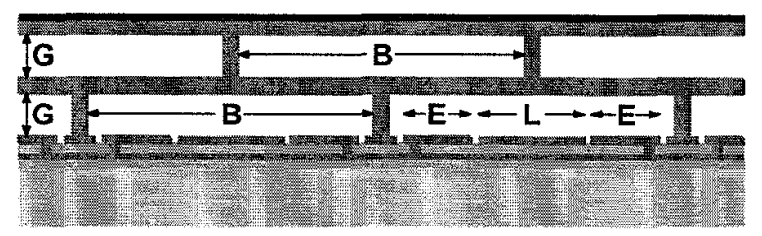

Figure 1. View of Polychromator grating beam structure in crosssection for unactuated state ( $B$ is the doubly-supported beam length, $E$ is the length of one actuating electrode, $L$ is the ground pad length, and $G$ is the lower and upper free-space gap).

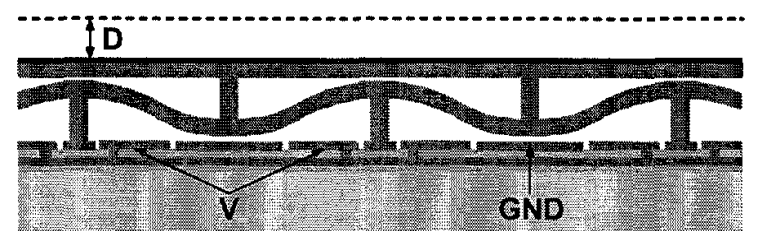

Figure 2. View of beam stmcture in cross-section for actuated state The lower beam undergoes bending, while the top mirror beam remains flat and deflects vertically $(D$ is the vertical displacement from the original position when voltage $V$ is applied to the actuating electrodes.



Figure 3. SEM of MEMS grating showing the double beam design, support structure, interconnects, and electrodes.

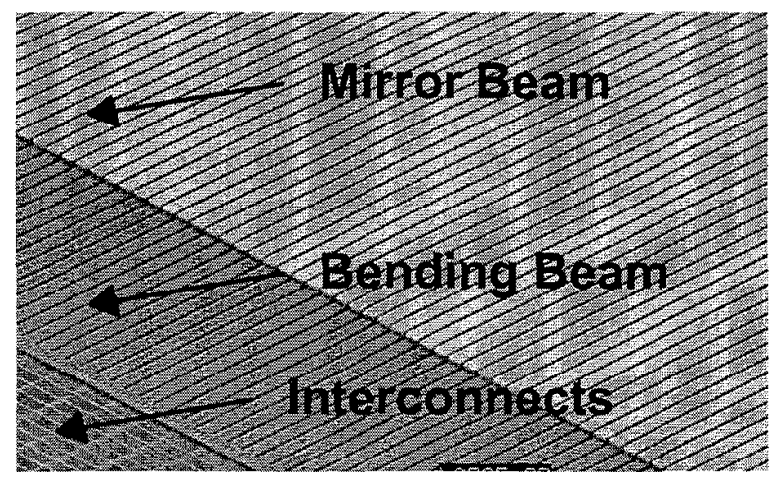

Figure 4. SEM of the edge of a MEMS Polychromator grating showing interconnects, lower bending beam, and upper mirror beam. Beam widths are $10 \mu$, and they extend $1 \mathrm{~cm}$ in length. 


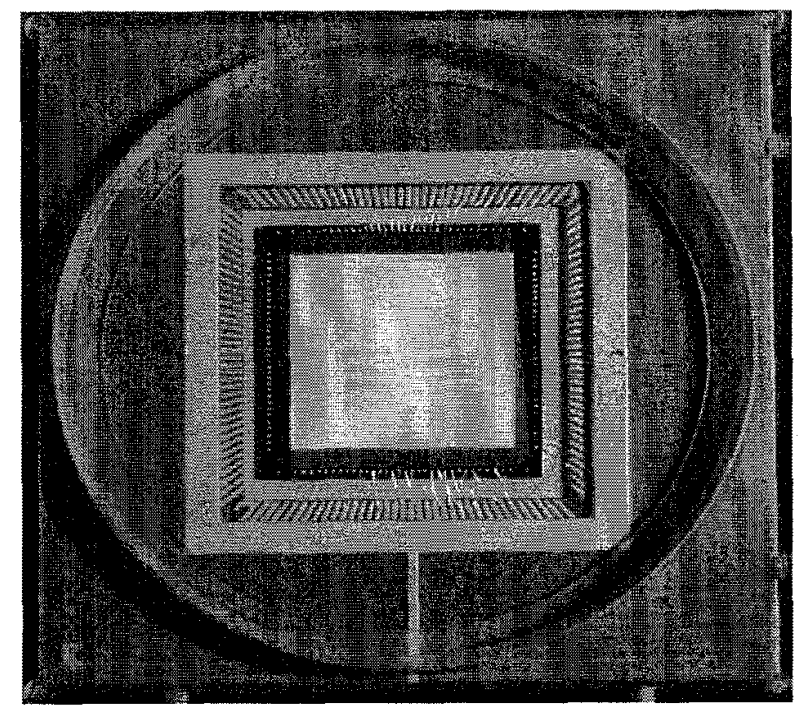

Figure 5. Packaged Polychromator die. Array is $1 \mathrm{~cm} \times 1 \mathrm{~cm}$.



Figure 6. Fringe shift of actuated beam using interference microscopy.

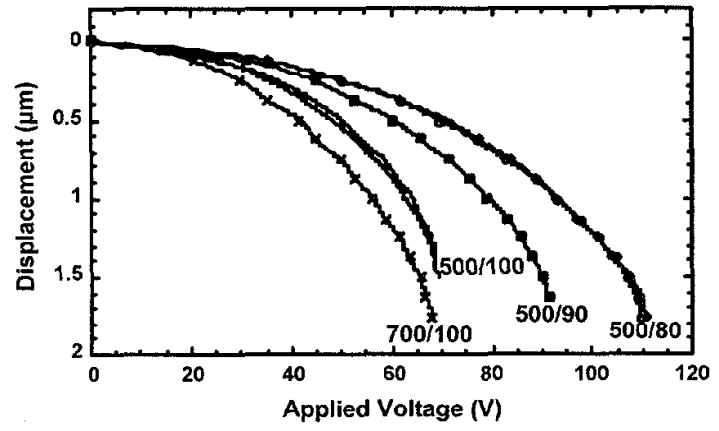

Figure 7. Displacement ( $\mu m$ ) versus actuating voltage $(V)$ for a variety of grating element designs (Numbers in the figure indicate the beam length to electrode length relationship $B / E$ in microns for each design).

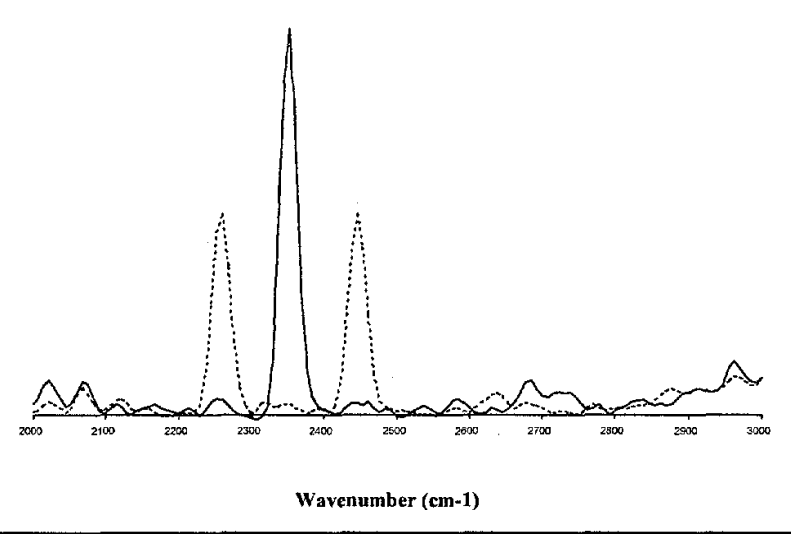

Figure 8. Spectral transmission of the Polychromator for the two grating profiles used for $\mathrm{CO}_{2}$ detection. The solid line spectrum matches the $\mathrm{CO}_{2}$ absorption at $2350 \mathrm{~cm}^{-1}$, while the doublebandpass dashed line spectrum samples an equal area outside the $\mathrm{CO}_{2}$ absorption. These spectra were measured with a Nicolet 750 FTIR spectrometer.

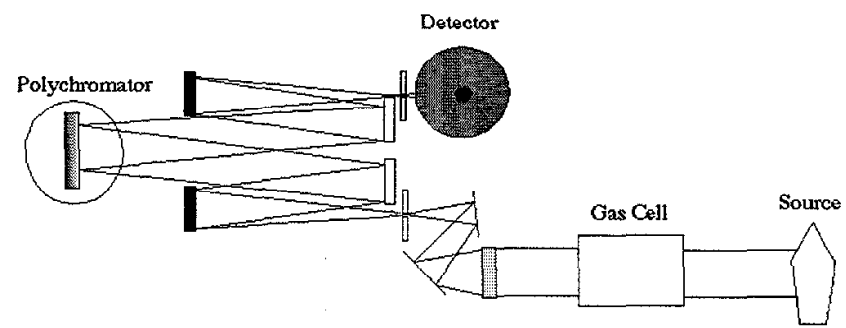

Figure 9. Diagram showing the optical configuration of the Polychromator-based correlation spectrometer. Further details are given in the text.

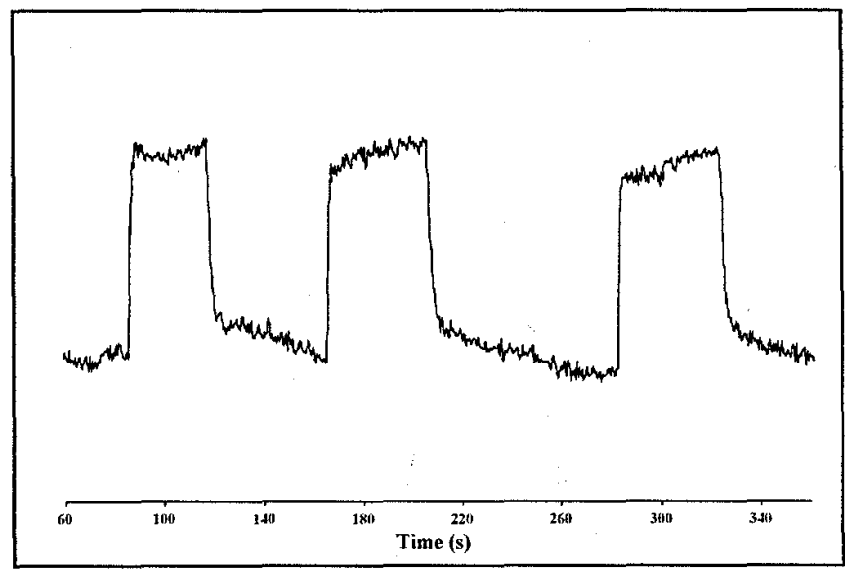

Figure 10. Response of the correlation spectrometer to $100 \mathrm{ppm}$ meters of $\mathrm{CO}_{2}$ admitted and purged from the gas cell 3 times. 